\title{
The differential representation of number and gender in Spanish
}

\section{Citation}

Fuchs, Zuzanna, Maria Polinsky, and Gregory Scontras. 2015. “The Differential Representation of Number and Gender in Spanish." The Linguistic Review 32 (4) (January 1). doi:10.1515/ tlr-2015-0008.

\section{Published Version}

10.1515/tlr-2015-0008

\section{Permanent link}

http://nrs.harvard.edu/urn-3:HUL.InstRepos:37108757

\section{Terms of Use}

This article was downloaded from Harvard University's DASH repository, and is made available under the terms and conditions applicable to Other Posted Material, as set forth at http:// nrs.harvard.edu/urn-3:HUL.InstRepos:dash.current.terms-of-use\#LAA

\section{Share Your Story}

The Harvard community has made this article openly available.

Please share how this access benefits you. Submit a story.

Accessibility 


\title{
Zuzanna Fuchs, Maria Polinsky, and Gregory Scontras
}

\section{The Differential Representation of Number and Gender in Spanish}

\begin{abstract}
This paper investigates the geometry of phi-features with a special emphasis on number and gender in Spanish. We address (i) whether number and gender evidence single- or multi-valued systems for their respective features, and (ii) whether number and gender are bundled together or split. Given the lack of consensus on these issues based on primary data, we approach these questions experimentally, using the phenomenon of agreement attraction: a situation in which ungrammatical sequences are perceived as grammatical when one of the NPs is erroneously identified as determining agreement. Our results indicate that number but not gender in Spanish is multi-valued and offer novel support in favor of an agreement model in which number and gender are in separte projections and are valued independently. We also offer some considerations concerning parametric variation in such valuation.
\end{abstract}

Keywords: feature geometry, number, gender, agreement, agreement attraction, Spanish

Zuzanna Fuchs, Maria Polinsky: Department of Linguistics, Harvard University

Gregory Scontras: Department of Psychology, Stanford University

\section{Introduction}

\subsection{Bundling and splitting in feature geometry}

Agreement is everywhere, which is probably why it is so hard to determine where exactly it is; researchers continue to debate about whether it is syntactic (e.g., Baker, 2008; Preminger, 2014), post-syntactic (e.g., Bobaljik, 2008), or distributed across several levels of representation (e.g., Wechsler and Zlatić, 2003). Another set of unresolved issues deals with the nature and representation of the features involved in agreement: phi-features. The classes of phifeatures are well known; despite the apparent variability of agreement, the recurrent features that it may track are constant: person, number, and gender. What remains less clear is the relationship of these features to each other 
and their hierarchical arrangement in agreement systems. These are the relationships that are often subsumed under the rubric of feature geometry.

This paper addresses feature geometry with respect to two out of three features, namely, number and gender. The choice of these two features is not arbitrary. The hierarchical position of person with respect to the other features is relatively well understood (cf. Harley and Ritter, 2002); it is also known that person features do not appear on non-verbal probes, which again separates it from the other features (Baker, 2008). Meanwhile, the relationship between gender and number is less clear. Assuming that both features are represented in syntax, there are two analytical possibilities, both proposed in the literature. According to one scenario, gender and number are always bundled together (cf. Ritter, 1993; Carstens, 2000, 2003); we will be referring to this approach as the bundling model. Under that model, all gender morphology is either hosted on the number head, as shown in (1), or is expressed on the specifier within a number phrase, as shown in (2). Either way, gender features do not project independently of number, and the valuation of gender presupposes a valuation of number. In other words, gender and number are bundled together.

$$
\begin{aligned}
& {\left[\text { NumP } \ldots\left[\operatorname{Num}_{\{\text {Number, Gender }\}}\right]\right]} \\
& {\left[\text { NumP } \mathrm{XP}_{\{\text {Gender }\}}\left[\operatorname{Num}_{\{\text {Number, Gender }\}}\right]\right]}
\end{aligned}
$$

The bundling model draws its empirical inspiration from the fact that languages regularly combine gender and number information; one rarely finds systems where the two features participate in agreement and yet are independent of each other. Furthermore, gender is lexically specified; a given noun belongs to gender $\mathrm{X}$ regardless of its syntactic position. The noun leaves the lexicon with a gender, and this gender persists throughout its use. Here gender stands apart from number, which is specified within a given eventuality: the number feature of a noun depends on its intended referent in a given use. Thus, number is tightly linked to event structure, the way that case is. Since gender is not directly linked to the event or argument structure, bundling models argue that it is desirable to have its representation in syntax mediated by another grammatical feature which is directly mapped into syntax. Such mediation is akin to representing animacy only indirectly via some other feature, for example via case. And finally, a strong argument for the bundling model is based on the gender of inanimate nouns. The gender of such nouns is uninterpretable, which in turn means that the gender projection cannot always have consistent semantic content. Elimination of semantically inconsistent projections is an important theoretical goal within the minimalist program (Chomsky, 1995), 
so eliminating an independent and semantically heterogeneous gender projection would result in a more parsimonious theory.

In the alternative, split model (Picallo, 1991; Carminati, 2005; AntónMéndez et al., 2002, a.o.), gender morphology hosted on a nominal stem heads its own projection (GenP). GenP is dominated by NumP (i.e., the source of number features/morphology), as schematized below:

$$
[\text { NumP }[\text { GenP ... }]]
$$

One of the major arguments in favor of the split model comes from the order of morphemes in nominal derivations. In those languages where number and gender morphology can be descriptively separated, the order is Stem-GenderNumber, as in the following Spanish examples:

$$
\begin{aligned}
& \text { a. [[libr]-[GenP O-] [NumP } \mathrm{G}]] \text { 'books' } \\
& \text { b. [[libr]-[GenP O-] [NumP } \varnothing]] \text { 'book' }
\end{aligned}
$$

Because it levels the hierarchical distinction between number and gender, the bundling model does not have a straightforward way of predicting the ordering shown here. That the split model derives such an order is a side effect of the simple feature geometry: number dominates gender. Furthermore, as syntactic theory has been moving away from the division of features into uninterpretable and interpretable, and toward giving more weight to feature valuation itself (Pesetsky and Torrego, 2007; Preminger, 2014), one of the otherwise strong theoretical arguments in favor of the bundling model (i.e., eliminating an uninterpretable gender projection) may be losing some of its heft.

In what follows, we present an attempt to take the debate between bundling vs. splitting out of purely theoretical considerations and ground it in the psychological reality of agreement itself, as applied to Spanish. The logic is as follows. On the widely accepted assumption that grammar and its parser are in an isomorphic relation (e.g., Phillips, 2013), observing the parser allows for the observation of the grammar. In other words, speakers' behavior in the parsing of agreement phenomena should stand as the proving ground for the theories that underlie the phenomena. Much of the work in such an approach centers around developing testable hypotheses about behavior on the basis of articulated theories of grammar. To this end, we turn now to further background on the theory of agreement features and their geometry. 


\subsection{Internal structuring of agreement features}

An evaluation of the two approaches to feature geometry sketched above presupposes knowledge of the way a feature is internally structured in a given language. Let us clarify this notion by analogy with the inflectional phrase (IP). In some languages, IP is represented by a tense phrase, in others, by an aspect phrase, and its language-specific organization can have morphosyntacic repercussions for clause structure. Likewise, the morphosyntactic structuring of gender and number cannot a priori be assumed to be the same across-theboard; it has to be evaluated in the context of a given language before the evaluation of bundling vs. split models in that language.

The internal structuring of number and gender can be represented in terms of feature specification. For example, in a gender system that divides all nouns into masculine and feminine, are both genders specified morphologically? And are they both specified semantically? Morphological specification can be directly observed; if one of the genders requires extra marking and the other does not, we can state that the former is morphologically specified and the latter, unspecified. Semantic specification can be operationally determined using the Greenbergian Taghlīb test (Greenberg, 1966; Sauerland et al., 2005)1: given a plurality of individuals, where some but not all have the specified (marked) property, only the unspecified form in a pair of features can be used to refer to that plurality. Let us illustrate the notion with an informal example. Should you receive an invitation to a party stating that you are welcome to bring guests, singular reference is included in the use of the plural. In other words, bringing only one guest would be in compliance with the invitation. However, an invitation stating that one is welcome to bring a guest does not include the plural reference with the use of the singular. Showing up with more than one friend would violate the letter of the invitation. This goes to show that in English, the singular is semantically specified as it restricts nominal reference to just singular individuals; the plural, however, is semantically unspecified, permitting reference to both singulars and plurals. But when it comes to the actual feature content (i.e., the possible values for number), we face competing pressures from semantics and morphology. The Taghlīb Test suggests a singular feature, but the morphological exponence of the plural suffix - $s$ suggests a plural feature. In fact, most theories of number posit that both features get

1 Greenberg (1966) uses this test and attributes it to Arab grammarians, giving it the Arabic name taghlīb 'dominance'. 
specified in the determination of nominal semantics and agreement, at least in English (cf. Sauerland, 2003; Scontras, 2013a,b).

When it comes to gender, we need to expand our sights beyond English. Many languages have gender systems, so there is no shortage of choices. Our choice of Spanish, the language we will focus on here, is motivated by several considerations. First, the structure of Spanish number is sufficiently similar to that of English, which allows for an effectual comparison between the two languages. Second, as we show below, the two genders of Spanish are distributed roughly equally, which is important in minimizing the potential imbalance between the genders due to statistical preferences. Third, there is debate surrounding the internal structure of Spanish gender. Some more practical considerations include the fact that there is already some experimental work on Spanish number and gender, which allows us to build upon existing findings. And finally, Spanish is widely spoken, which facilitates access to a large subject pool.

Since most of the issues surrounding number and gender geometry arise from the consideration of inanimate nouns, our work will be limited to these nouns. A number of researchers have advocated separating the gender of animates and the gender of inanimates, distinguishing them as high and low gender, respectively (cf. Kramer, 2009, 2013; Bobaljik and Zocca, 2011). Assuming this division is on the right track, our focus is only on the low, lexical gender. Still, we anticipate that future work could include high gender; we return to this point in section 6 .

The remainder of the paper is structured as follows. In section 2 we present a brief overview of the relevant empirical data in Spanish; we address the nature of number and gender oppositions and in particular present a summary of the seminal paper by Harris (1991), who argues that Spanish gender is a singlevalued opposition. In section 3 we introduce the experimental work applied in this study and present its main results. Section 4 establishes the internal structuring of Spanish number and gender in light of the experimental results. Based on this structuring, we compare the bundling and the split models of Spanish phi-features in section 5. Section 6 concludes with a discussion of our results in relation to languages other than Spanish.

\section{Background: Spanish number and gender}

Agreement in Spanish is rampant: determiners, adjectives, and participles must all agree with a head noun in both number and gender. In addition, 
this agreement in number and gender is maintained in anaphors. To better understand the features involved in this agreement, we start with a fuller discussion of Spanish number, then turn to gender.

\subsection{Number}

Spanish has two numbers: singular and plural. Much like in English, the plural is morphologically marked with the suffix $-s$, while the singular appears morphologically bare.
a. la manzana 'the apple'
las manzanas 'the apples'
b. el plátano 'the banana'
los plátanos 'the bananas'

In terms of the semantic specification of number, Spanish is again similar to English. Consider the following examples:
a. el certificado médico para la tenencia de animales the certificate medical for the ownership of animals peligrosos dangerous 'the medical certificate for the ownership of dangerous animals'
b. el certificado médico para la tenencia del animal peligroso the certificate medical for the ownership of animal dangerous 'the medical certificate for the ownership of a dangerous animal'

Example (6-a) implies that one may own several dangerous animals, or just one; as in English, singular reference is included in the use of the plural. On the other hand, (6-b) clearly restricts ownership to no more than one dangerous animal; plural reference is not included in the use of the singular. These facts suggest that in Spanish, the singular is semantically specified, while the plural is semantically unspecified. Thus, with the internal composition of number, Spanish faces competing pressures from semantics and morphology. The Taghlīb Test suggests that the singular is semantically specified, but morphology specifies the plural with $-s$. As in English, these competing pressures suggest that both singular and plural number features are equally available. 


\subsection{Gender}

Spanish has two genders, masculine and feminine, which are distributed approximately equally in the nominal lexicon: masculine $52 \%$, feminine $45 \%$ (Bull, 1965).2 With respect to semantic specification, only the feminine is semantically specified. Consider los padres, which is ambiguous between 'parents' and 'fathers'; on the first reading, feminine reference is included in the use of the masculine ('parents' often references classes of mixed gender). In contrast, las madres 'mothers' can only refer to a plurality of women, but not to parents of both genders. In a different realization of the same pattern, if two or more nouns of both genders are coordinated, the agreeing adjective has to be the masculine:

El libro y la pintura son preciosos $/ *^{*}$ preciosas

[the book].M and [the painting].F are expensive.M.PL/expensive.F.PL

'The book and the painting are expensive.'

Whether or not we posit an alignment between the morphological and semantic specification of Spanish gender depends on the analysis of nominal morphology that gets assumed. For example, if we take $-o$ and $-a$ to be the morphological realization of masculine and feminine gender, respectively, then both genders are equally specified morphologically. However, this morphological analysis of gender has been questioned, most notably by Harris (1991). He notes that neither word marker (i.e., $-o$ and $-a$ ) is exclusively associated with a particular gender. The typically masculine word marker - $o$ can also appear on feminine nouns (e.g., la mano 'the hand'), nouns that can be masculine or feminine (e.g., el/la testigo 'the witness'), as well as words not associated with a gender at all (e.g., the preposition dentro 'inside'). Similarly, the word marker $-a$, which is typically associated with feminine nouns, can also mark masculine nouns (e.g., el día 'the day'), nouns that can be masculine or feminine (e.g., el/la turista 'the tourist'), and words associated with neither gender (e.g., the preposition afuera 'outside'). In addition, coordinate noun phrases with ostensibly different gender values on the subconstituents determine masculine agreement, as in (7) above, and neologisms are assigned masculine gender regardless of their morphological shape - for instance, the preposition para 'for' is inherently genderless, but when mentioned metalin-

2 The three percent discrepancy in the numbers is due to the presence of epicene, or gender-neutral nouns. Bull's numbers point to a slight predominance of the masculine, but in practical terms the two genders are as balanced as it ever gets. 
guistically it will also determine masculine agreement. Based on the lack of a consistent mapping between specific endings and genders, we lack clear arguments concerning the morphological specification of Spanish gender. Based on the facts just sketched, Harris (1991) argues that only the feminine in Spanish gets specified morphologically, which aligns the morphological and semantic specification of gender in Spanish and provides support for his claim that only the feminine feature gets specified: in the absence of feminine, nouns appear with default (i.e., masculine) morphology and receive default semantics. In other words, what is traditionally described as masculine gender is simply the lack of a feminine feature; there is no masculine gender feature in Spanish.

The opposite view, according to which Spanish gender is multi-valued, has also received support. According to Roca (1989), Domínguez et al. (1999), and Alarcón (2006), the surface facts of Spanish indicate equal presence of a feminine and a masculine gender features in Spanish. The multi-valued account is designed to reflect the morphology of determiners, where each of the genders enjoys equal representation, and the marking on determiners is subtractive for the masculine only in the indefinite singular ( un vs. una); in all other subsystems, both genders have equally visible morphology on their determiners: el/la, los/las, unos/unas. Thus, under the multi-valued approach, both genders are specified morphologically as a result of the availability of two gender features, masculine and feminine.

\subsection{Previous experimental work}

To date, our predecessors in the empirical investigation of the Spanish agreement system have restricted themselves to different methodologies or to considering the relationship between number and gender without first investigating their feature specification. We review this work below.

In a production study of the behavior of Spanish number and gender, which builds on Antón-Méndez (1999), Antón-Méndez et al. (2002) presented speakers with a predicative adjective as well as a complex noun, designing the complex nouns such that each contained a distractor noun phrase that could erroneously control agreement (more on this in our discussion of agreement attraction below). They then asked the speakers to reproduce the complex noun and complete the sentence using the adjective they had been shown. The researchers measured the frequency of number errors and gender errors made in agreement between the head noun and the adjective, as well as the frequency of number errors in agreement between the head noun and verb. 
The authors focused on conditions in which the distractor noun mismatched the head noun in both number and gender. If number and gender are dependent (i.e., bundled together), they claim, all errors in agreement between the adjective and the head noun that involve number should also involve gender, and vice versa (what Antón-Méndez et al. refer to as "combined errors"). If they are independent, however, then laws of probability suggest we should observe far lass combined errors than either number errors or gender errors in agreement.

The results of the experiment conducted by Antón-Méndez et al. showed that the frequency of combined errors was much smaller than the frequency of just number errors or just gender errors. In fact, a comparison of the observed probabilities of number, gender, and combined errors was consistent with a model in which the probability distributions of number errors and gender errors were independent of each other. The authors took the evidence as support for a model in which number valuation and gender valuation are independent, which in turn argues in favor of an analysis in which number and gender are projected independently - a split model.

However, Antón-Méndez et al. found that, while gender and number may not be dependent, they are sensitive to each other. Their results show that speakers were more likely to make a gender agreement error between the head noun and the adjective if the head noun and distractor noun were both singular. Similarly, speakers were more likely to make a error in number agreement if the head noun and distractor noun were of the same gender. The result is an apparent contradiction: Valuation of number and valuation of gender are independent, yet seemingly sensitive to one another. The authors discuss this paradox, stating that the observed correlation between number and gender is inconsistent with the independence of the two features. They also suggest that these effects may be the result of a monitoring system (Levelt, 1989) that is more likely to allow an error to go unnoticed if two expressions are more similar to each other in agreement features.

Antón-Méndez et al. present results with somewhat conflicting implications for the relationship between number and gender. On the one hand, the probability distributions of number, gender, and combined errors suggest that number and gender are independent, as in a split model. On the other hand, the sensitivity of the two features to each other contrasts with this claim and may be more compatible with a bundling model. On the basis of their results, then, we are unable to properly adjudicate between a bundling model and a split model.

The work of Antón-Méndez et al. is complemented by other experimental work on the interaction of number and gender agreement errors in Spanish. 
Vigliocco et al. (1996) conduced a similar production study to the one described above, but added a semantic variable for consideration. The goal of their experiment was to determine whether distributivity effects can play a role in number agreement errors. The preambles they presented to the participants of their completion study also had a head noun and a distractor noun nested within a modifier, but the plurality of one of these nouns was designed to have a possible effect on the conceptual plurality of the other. Take, for instance, the label on the bottles. While the label is grammatically singular, the plural noun in the modifier on the bottles may lead speakers to understand the label as referring to a plurality of labels (this is referred to as a 'distributivity' effect). Vigliocco et al. found that semantics, specifically distributivity effects, does play a role in number agreement errors: Spanish speakers were more likely to make an agreement error if they perceived the head noun as possibly semantically plural based on the plurality of the distractor noun.

Alcocer and Phillips (2009) contribute to the discussion of subject-verb agreement in Spanish by making a distinction between agreement errors in sentences in which the agreeing verb is an auxiliary (8) and sentences in which the agreeing verb is a main verb (9). As in both examples below, the agreeing noun and verb were both located in a relative clause, with a non-local distractor noun located outside of the relative clause ('the note' in both examples below).

la nota que la chica va a escribir... the note COMP the girl go.3SG to write.INF 'the note that the girl is going to write'

(9) la nota que la chica escribió... the note COMP the girl write.3SG.PST 'the note that the girl wrote'

In sentences with noun-auxiliary agreement, (8), they found that speakers were more likely to judge a grammatical sentence to be ungrammatical if the nonlocal distractor was plural. In sentences with noun-main verb agreement, (9), they found no such effect. Based on these results, Alcocer and Phillips suggest that there is a difference between noun-auxiliary agreement and noun-main verb agreement. They conducted a similar experiment on English and found opposite results: English speakers were more likely to judge an ungrammatical sentence to be grammatical if the distractor noun was plural. By comparing results across the two languages, Alcocer and Phillips concluded that less marked verb forms are more susceptible to interference from a distractor noun. For instance, the English see is unmarked as compared to the third-person 
singular sees and is therefore more susceptible to an agreement error if a plural distractor noun is present. In Spanish, the third-person singular va 'go' is the less marked form (as compared to the third-person plural van 'go') and is more susceptible to interference from a plural distractor noun. Alcocer and Phillips thus demonstrate that properties of the agreeing verb may also have an effect on the likelihood of an agreement error in the presence of distractors.

What is missing from this not insubstantial body of experimental work on agreement in number and gender in Spanish is a systematic approach to observing the interaction between number and gender, one that tracks the feature specification while investigating its geometry. Such an approach would recognize that understanding the relationship between these two features first requires an understanding of their respective feature content. The experiment we present next aims to do just that.

\section{Experiment}

\subsection{Goals}

The goal of the experimental work presented below is to determine whether there is evidence for the bundling vs. split model of gender and number as applied to Spanish. To gain this kind of evidence we need to explore agreement violations in each of the features and more specifically to put number and gender in competition with each other. If Spanish resolves this competition differently for gender than for number, then we have evidence in favor of the split model. If gender and number are resolved together, we have evidence for bundling.

By holding number agreement constant and manipulating gender agreement in a context in which there exists a potential for conflict between features, we can observe how the two potential gender values in Spanish interact with each other and thus gain insight to the feature content of Spanish gender. By holding gender agreement constant but creating conflict in number agreement, we can do the same for the feature content of number. Simultaneous manipulation of number and gender will similarly give insight into the interaction between number and gender and therefore into the relationship between the two features.

While ours is not the first study investigating the relationship between number and gender features, as well as the features themselves, it is the first to investigate these issues simultaneously and within a comprehension set- 
ting. Previous studies have either focused on only one of the features that we investigate and how this feature behaves in agreement attraction contexts, or they have investigated the potential differences between noun-verb agreement or noun-predicative adjective agreement. Our study also increases the syntactic distance between the source noun and agreement target in order to tax the memory and thus strengthen any possible effects, making them more apparent.

\subsection{An excursus on agreement attraction}

A context where agreement features are forced to compete with each other is available when they are represented on different goals which are, licitly or illicitly, accessible to the same probe. Agreement attraction is one such context. In cases of agreement attraction, a feature-seeking probe fails to match the grammatical features of the appropriate goal and instead realizes the grammatical features of a distractor, an expression that is usually closer to the probe in linear order. Consider an example from English as an illustration:

(10) The book that was left on the table by the girls have a blue cover.

As a speaker produces this sentence, s/he stores the book in memory as the subject of the sentence, looking for a verb with which it must agree in person and number. As the sentence proceeds, the memory is taxed by the introduction of several other DPs (the table, the girls). The agreement features of the original subject enter into conflict with the other information that the speaker must store in memory, including the features of these interfering DPs, even if they are embedded under a prepositional head. This may result in incorrect agreement where the features of the book have been replaced in memory by the features of the girls. Agreement attraction is particularly powerful in single-valued systems; if only one feature value is visible and the other is simply an absence of a feature value, the visible feature should strongly drive attraction (see Bock and Cutting 1992; Franck et al. 2002, 2006 on number attraction). For this reason, it is crucial to understand the content of feature values in Spanish gender and number before we move into the comprehension of attraction errors.

Cases of agreement attraction have been experimentally studied in various languages (English: Bock and Miller, 1991; Bock and Eberhard, 1993; Vigliocco et al., 1996; Vigliocco and Nicol, 1998; Bock et al., 2012; Spanish: Vigliocco et al., 1996; Antón-Méndez, 1999; Antón-Méndez et al., 2002; Al- 
cocer and Phillips, 2009; Italian: Vigliocco et al., 1995; Vigliocco and Franck, 1999; French: Vigliocco et al., 1996; Dutch: Bock et al., 2001; Hartsuiker et al., 2003; German: Hartsuiker et al., 2003; Russian: Lorimor et al., 2008, a.o.), testing whether there is an asymmetry between different values of features in triggering agreement errors. In particular, previous studies looked at errors involving number (Bock and Miller, 1991; Bock and Eberhard, 1993; Vigliocco et al., 1996; Bock et al., 2012; Alcocer and Phillips, 2009; Vigliocco et al., 1995; Hartsuiker et al., 2003; Bock et al., 2001; Vigliocco and Nicol, 1998), gender (Vigliocco and Franck, 1999), or both gender and number (AntónMéndez, 1999; Antón-Méndez et al., 2002; Lorimor et al., 2008). We can build on this substantial body of work and put the phenomenon of attraction to use in exploring the difference between bundling and split approaches. We make minimal assumptions concerning the actual mechanism of attraction, noting merely the diverging predictions of bundling vs. split approaches, which we turn to next.

\subsection{Predictions}

Based on the discussion of their respective feature content above, we can make certain predictions concerning the feature content of Spanish number and gender. If a given feature value is specified, then when placed into conflict with another feature value, we should see grammaticality effects. That is, speakers will distinguish between sentences in which there are no agreement errors pertaining to the specified feature value, and ones in which there are agreement errors pertaining to the specified feature value - they should rate the former significantly higher than the latter. Consider an example:
a. El niño considera la noticia en las
the boy consider.3SG the news item.F.SG in the
revistas terriblements aburrida.
magazines.F.PL terribly boring.F.SG
'The boy considers the news item in the magazines to be terribly boring.'
b. *El niño considera la noticia en las
the boy consider.3sG the news item.F.SG in the
revistas terriblements aburridas.
magazines.F.PL terribly boring.F.PL

The first of the two sentences is grammatical, but the second has an error in number agreement - escritas 'written' is plural, but should be singular in 
agreement with la carta 'the letter'. If singular is a specified feature value in Spanish, then grammatically correct sentences like (11-a) should consistently be rated higher than sentences with an error in agreement in the singular, like the sentence in (11-b).

It follows that if a feature is multi-valued, that is, has two specified values, both of them should exhibit grammaticality effects. If, however, a feature is single-valued, only one of the two potential values will exhibit grammaticality effects. This leads us to make the following predictions:

Prediction 1: If number is multi-valued, both singular and plural will show grammaticality effects.

Prediction 2: If number is single-valued, either singular or plural (but not both) will show grammaticality effects.

Prediction 3: If gender is multi-valued, both masculine and feminine will show grammaticality effects.

Prediction 4: If gender is single-valued, either masculine or feminine (but not both) will show grammaticality effects.

Having settled the feature content of number and gender, we can then consider whether the data indicates the bundling or the split model of number and gender feature classes. Recall that if number and gender and bundled, then they ought to be valued simultaneously. This suggests the number and gender features of a noun should determine agreement together, at the same time. When an incorrect noun enters into agreement with an adjective, both its number and gender features should agree with the number and gender features of the adjective. Building on our example in (11), consider the following ungrammatical sentences:

$$
\begin{aligned}
& \text { a. }{ }^{*} \text { El niño considera la noticia en los } \\
& \text { the boy consider.3sG the news.item.F.SG in the } \\
& \text { periódicos terriblemente aburridos. } \\
& \text { magazines.M.PL terribly } \quad \text { boring.M.PL }
\end{aligned}
$$

b. *El niño considera la noticia en las the boy consider.3sG the news.item.F.SG in the revistas terriblemente aburridas. magazines.F.PL terribly boring.F.PL Intended: 'The boy considers the news item in the magazines to be terribly boring.' 
Both of the above sentences are grammatically incorrect, but in each of them the local noun has entered into agreement with the adjective, which may lead to an illusion of grammaticality (i.e., attraction). If number and gender are projected and valued together, as in a bundling approach, then when the probe (incorrectly) gets a feature (e.g., number) from the local noun, it should get the other feature (e.g., gender) as well. In other words, agreement attraction in one feature ought to precipitate agreement attraction in the other feature, with the result that both of the above sentences should be rated equally high (or equally low).

If, however, number and gender are split, then they are projected and valued independently, and agreement attraction in number can proceed independently of agreement attraction in gender. This means that, all factors being equal, a violation in gender agreement may be judged higher or lower than a violation in number agreement. Of course, each type of violation may also be judged roughly the same, but crucially, they are evaluated on their own, without a connection to the other type of violations. Furthermore, if the two features are independent of each other, we can expect that a violation in both of them would be more offensive to a comprehender than a violation in just one feature. This expectation is based on the observation that the more grammatical constraints violated, the higher the degree of degradation (cf. Kluender, 2004).3 Applying that logic, we expect that the violation in (12-a), where both the gender and the number of the head noun are mismatched, should be rated lower than (12-b), where only the number is mismatched. Thus, under a split model, (12-a) should receive a lower rating.

To summarize the bundling vs. split predictions:

Prediction 5: If number and gender are bundled, agreement attraction in one feature should lead to agreement attraction in the other feature.

Prediction 6: If number and gender are split, agreement attraction in one feature need not lead to agreement attraction in the other feature.

We can now turn to the design of the experiment used to test these predictions.

3 The issue of cumulative effects of violations is not open-and-shut, and needs to be evaluated on a case by case basis. It is possible that some violations are more "costly" than others, and their hierarchical arrangement may call for an Optimality Theory-type ranking (see Warren and Gibson, 2002 for a discussion of such an approach to processing). However, in comparing phi-feature violations, we can reasonably assume that they are comparable in visibility, which would entail a cumulative effect when both features are mismatched. 


\subsection{Participants}

We recruited 126 participants through Amazon.com's Mechanical Turk crowdsourcing service. Subjects were compensated for their participation. We restricted IP addresses of subjects to just those within the United States in an attempt to control for language background, specifically familiarity with European vs. Latin American Spanish vocabulary. On the basis of a short demographic questionnaire that preceded the experiment, we determined whether subjects were native speakers.

\subsection{Design}

Our goal of simultaneously evaluating number and gender agreement errors, with intervening material between the goal and the probe, constrained the possible syntactic frames we could use in the design of our stimuli. We needed a configuration in which a noun intervenes between the source and target of agreement, and in which all of the agreeing elements inflect for both gender and number. Such a configuration is observed in argument small clauses such as the one below:

$$
\begin{aligned}
& \text { Encuentro estos libros muy interesantes. } \\
& \text { find.PRs.1SG these books.M.PL very interesting.M.PL } \\
& \text { 'I find these books very interesting.' }
\end{aligned}
$$

We rely on the existing syntactic analysis of such small clauses (Contreras, 1987; Jiménez-Fernández and Spyropoulos, 2013), and assume that they have the following structure, representing (13): 
(14) Small clause structure:

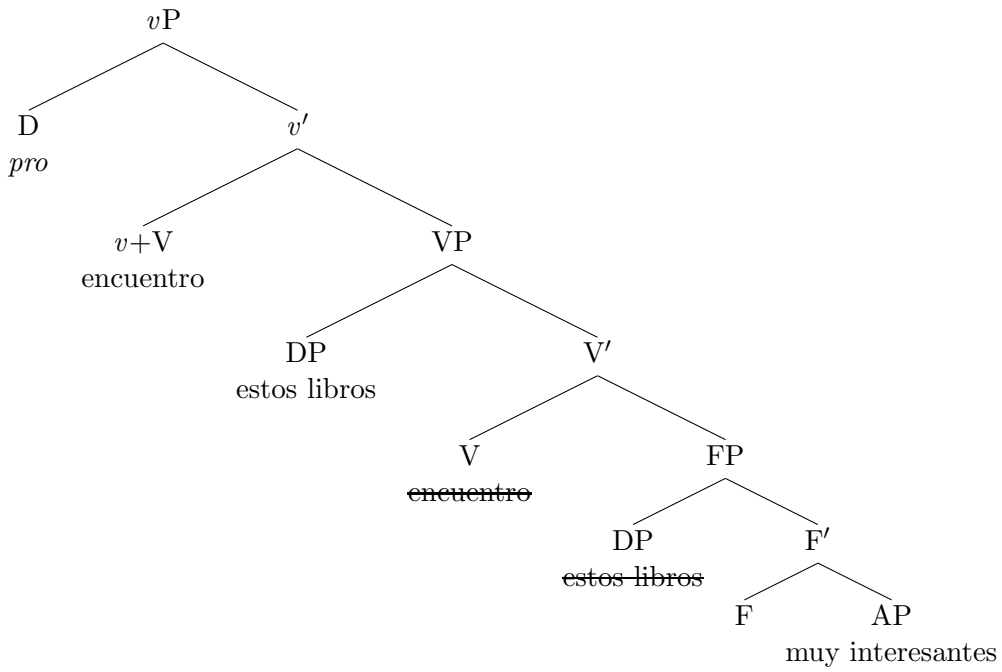

Zooming in on the small clause portion of the example in question, we observe number and gender agreement on the predicative adjective, thus:

Small clause feature valuation:

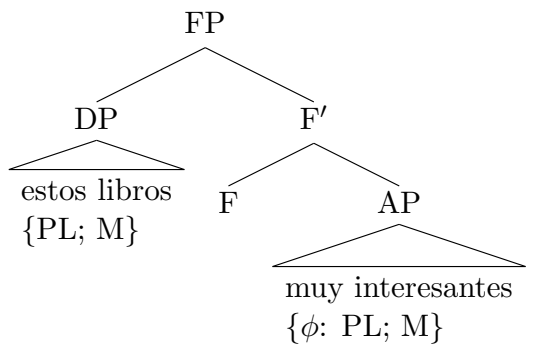

The goal DP can be made heavier with the introduction of a prepositional phrase modifying it, thus:

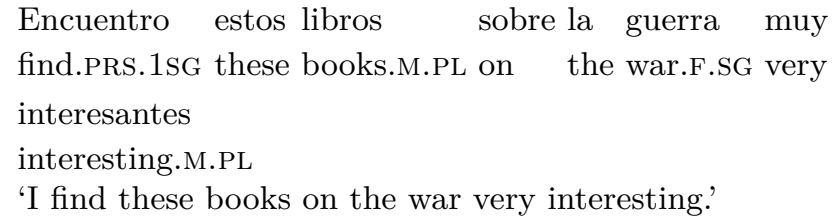

This is the precisely the configuration which would allow us to investigate agreement errors and attraction by systematically manipulating the features 
of the two nouns and the adjective; schematically it can be represented as follows:

$$
\text { (subject) verb NP1 prep NP2 adv ADJ ... }
$$

Only a handful of verbs in Spanish embed small clauses. In addition to encontrar 'find', our stimuli featured considerar 'consider' and ver 'see' as the matrix verb. We made a distinction between matrix predicates which subcategorize for small clauses, and those that can co-occur with non-subcategorized small clauses or secondary predicates (i.e., depictives), such as dejar 'leave' (Contreras, 1987; Demonte, 1988). In the experiment reported below, we included both subtypes to test if any differences between subcategorized and non-subcategorized small clauses would appear in agreement. In this paper, we will not discuss the comparison between the two types, but, in a nutshell, there was no significant differences between them with respect to agreement.

Stimuli were recorded by an adult male native speaker of Spanish from Colombia. To avoid possible phonetic cues to ungrammaticality, only the grammatical versions of our items were recorded. We then split the sentence recordings at the adverb and spliced each onset ((subject) verb NP1 prep NP2... ) with the four possible adjective completions (ADJ.M.SG, ADJ.M.PL, ADJ.F.SG, ADJ.F.PL). All stimuli were spliced together in this manner, even the fully grammatical variants.

Fifteen items were created, with 64 versions of each. Within each item, we manipulated the number (SG vs. PL) and gender (M vs. F) of NP1, NP2, and ADJ; this manipulation yields 64 sentences: 2 NP1-NUM x 2NP1-GEN $\mathrm{x}$ $2_{\mathrm{NP} 2 \text {-NUM }} \times 2_{\mathrm{NP} 2 \text {-GEN }} \times 2_{\mathrm{ADJ}-\mathrm{NUM}} \times 2_{\mathrm{ADJ}-\mathrm{GEN}}=64$.

Given that we are interested in the behavior of grammatical gender, that is, the gender a noun leaves the lexicon specified for, our gender manipulation on nouns required the use of different lexical items for masculine vs. feminine values. Within an item, we matched the meaning of these nouns as closely as possible (as with el armario 'the cupcoard' and la alacena 'the cupboard' in (18)); a full list of our experimental stimuli can be found online (Scontras et al., 2013).

A crucial aspect of the design of our stimuli concerns the way in which we effect agreement errors: only when NP1 and ADJ agree in both number and gender will the sentence be grammatical; all other versions of an item will involve a mismatch in the features of NP1 and ADJ and therefore be ungrammatical. In (18), we give two grammatical versions of one of our items. 
a. Maria considera el platillo en el armario

Maria consider.PRS.3SG the plate.M.SG in the cupboard.M.SG elegamente decorado pero prefiere recoger las elegantly decorated.M.SG but prefer.PRS.3SG collect.INF the teteras teapots.

b. Maria considera la bandeja en la alacena Maria consider.PRS.3SG the tray.F.SG in the cupboard.F.SG elegamente decorada pero prefiere recoger las elegantly decorated.F.SG but prefer.PRS.3SG collect.INF the teteras teapots 'Mary considers the tray in the cupboard to be elegantly decorated, but she prefers collecting teapots.'

We have assumed that NP1 (in bold) must agree with ADJ (in italics); when the features of the two do not match, ungrammaticality results. Note, however, that correctly identifying grammaticality on this basis within our items requires that there is no parse of the sentence under which ADJ intentionally agrees with and modifies NP2 (underlined) and not NP1. Were NP2 to agree with ADJ, its features (an not those of NP1) would determine grammaticality.

To rule out this unintended parse of our stimuli (under which ADJ modifies NP2), we ran a separate norming study on all of our items. 60 participants who did not take part in the critical study rated the likelihood that NP2 (and not NP1) gets modified by ADJ on a five-point scale. Subjects consistently rated this parse low (average rating: 2 out of 5; for the full set of results see Scontras et al., 2013); on the basis of these low ratings we conclude that the unintended parse is highly unlikely, if not unavailable. We furthermore conclude that our agreement error manipulation is sound: the features of NP1 determine the grammaticality of the sentence.

Subjects took the experiment online using the web-based experiment platform ExperigenRT (Becker and Levine, 2010; Pillot et al., 2012), which allowed for the measurement of response times to our auditory stimuli. After filling out a short demographic questionnaire, subjects were presented with one version of each of the fifteen items, together with thirty fillers; stimuli were presented in a random order. Subjects provided acceptability ratings for the sentences they heard on a scale from 1 (completamente inaceptable 'completely unacceptable') to 5 (completamente aceptable 'completely acceptable').

Only responses from native speakers of Spanish were analyzed. We evaluated nativeness on the basis of two demographic questions: What was the first 
language you learned? and What is the language you speak most at home? Subjects who provided Spanish as answers to both questions were classified as native; data from 50 subjects was included in the analysis.

\subsection{Results}

Given the many factors at play in the design of our experiment, analyzing their contributions all at once would yield uninterpretable results. We therefore split the analyses into five parts: we begin by analyzing number features, looking at their effect on singular and then plural head nouns; we then shift to gender features, looking at masculine and then feminine head nouns. Finally, we compare potential attraction conditions.

SINGULAR HEAD NOUN. To avoid possible effects of gender mismatches on our investigation of number, we hold gender constant across all three of NP1, NP2, and ADJ. That is, either all three are masculine or all three are feminine. Keeping NP1 singular, we have four possibilities for the number features of NP2 and ADJ as in Table 1, which reports average ratings for the four resulting conditions.

Table 1. Average ratings given to singular head noun conditions

\begin{tabular}{ccc|cc}
\hline NP1-NUM & NP2-NUM & ADJ-NUM & Rating & SE \\
\hline SG & PL & PL & 3.92 & 0.16 \\
SG & SG & PL & 3.47 & 0.17 \\
SG & PL & SG & 3.83 & 0.17 \\
SG & SG & SG & 4.16 & 0.14 \\
\hline
\end{tabular}

We fit a mixed logit model predicting response by NP2-NUM and ADJNUM, as well as their interaction; the model included random intercepts for subjects and items, as well as random slopes for NP2-NUM and ADJ-NUM grouped by subject and by item. Here and in all of the analyses that follow we trimmed the data on the basis of response time to within two standard deviations of the mean. We find a marginal effect of $\operatorname{ADJ}-\mathrm{NUM}\left(\chi^{2}(1)=\right.$ 2.87, $\mathrm{p}=0.060)$ : grammatical sentences in which ADJ agrees with NP1 in the singular are rated higher than ungrammatical sentences with a plural ADJ. We also find a significant interaction between NP2-NUM and ADJ-NUM $\left(\chi^{2}(1)=5.62, \mathrm{p}<0.05\right)$ : sentences in which both NP2 and ADJ are plural 
are rated higher.

PLURAL HEAD NOUN. As in our analysis of singular head nouns, we keep gender features constant across NP1, NP2, and ADJ: all are either masculine or feminine. Average ratings for the four conditions with a plural head noun are given in Table 2.

Table 2. Average ratings given to plural head noun conditions

\begin{tabular}{ccc|cc}
\hline NP1-NUM & NP2-NUM & ADJ-NUM & Rating & SE \\
\hline PL & PL & PL & 4.18 & 0.15 \\
PL & SG & PL & 4.10 & 0.17 \\
PL & PL & SG & 3.70 & 0.15 \\
PL & SG & SG & 3.77 & 0.18 \\
\hline
\end{tabular}

We fit a mixed logit model predicting response by NP2-NUM and ADJNUM, as well as their interaction; the model included random intercepts for subjects and items, as well as random slopes for NP2-NUM and ADJ-NUM grouped by subject and by item. Here we find only a significant effect of ADJ-NUM $\left(\chi^{2}(1)=4.53, \mathrm{p}<0.05\right)$ : grammatical sentences in which ADJ agrees with NP1 in the plural are rated higher than ungrammatical sentences with a singular ADJ.

MASCULINE HEAD NOUN. Shifting our focus to the effects of gender agreement errors, we begin by analyzing responses to masculine head nouns. To avoid possible effects of number mismatch, now we keep the values for number constant across NP1, NP2, and ADJ: all three are either singular or plural. The four possible values for NP2 and ADJ gender are given in Table 3, in which we report average ratings for masculine head nouns.

Table 3. Average ratings given to masculine head noun conditions

\begin{tabular}{ccc|cc}
\hline NP1-GEN & NP2-GEN & ADJ-GEN & Rating & SE \\
\hline M & F & F & 3.77 & 0.18 \\
M & $\mathrm{M}$ & $\mathrm{F}$ & 3.47 & 0.19 \\
M & $\mathrm{F}$ & $\mathrm{M}$ & 4.31 & 0.13 \\
$\mathrm{M}$ & $\mathrm{M}$ & $\mathrm{M}$ & 4.11 & 0.15 \\
\hline
\end{tabular}


We fit a mixed logit model predicting response by NP2-GEN and ADJGEN, as well as their interaction; the model included random intercepts for subjects and items, as well as random slopes for NP2-GEN and ADJ-GEN grouped by subject and by item. We find a only a significant effect of ADJGEN $\left(\chi^{2}(1)=8.22, \mathrm{p}<0.01\right)$ : grammatical sentences in which ADJ agrees with NP1 in the masculine are rated higher than ungrammatical sentences with a feminine ADJ.

FEMININE HEAD NOUN. In Table 4 we present average ratings for the four conditions in which the NP1 is feminine and all three of NP1, NP2, and ADJ match for number.

Table 4. Average ratings given to feminine head noun conditions

\begin{tabular}{ccc|cc}
\hline NP1-GEN & NP2-GEN & ADJ-GEN & Rating & SE \\
\hline F & F & F & 4.23 & 0.14 \\
F & $\mathrm{M}$ & $\mathrm{F}$ & 4.34 & 0.15 \\
$\mathrm{~F}$ & $\mathrm{~F}$ & $\mathrm{M}$ & 3.89 & 0.17 \\
$\mathrm{~F}$ & $\mathrm{M}$ & $\mathrm{M}$ & 4.00 & 0.14 \\
\hline
\end{tabular}

We fit a mixed logit model predicting response by NP2-GEN and ADJGEN, as well as their interaction; the model included random intercepts for subjects and items, as well as random slopes for NP2-GEN and ADJ-GEN grouped by subject and by item. We found no significant effects.

Attraction COMPARisons. To evaluate possible additive grammaticality effects, and thus test bundling vs. split approaches to feature geometry, we further consider conditions with either one or two possible agreement errors, as in Table 3 .

Table 5. Potential attraction conditions with feminine head nouns

\begin{tabular}{ccc|cc}
\hline NP1 & NP2 & ADJ & Rating & SE \\
\hline F.SG & F.PL & F.PL & 4.26 & 0.16 \\
F.SG & M.PL & M.PL & 3.68 & 0.28 \\
\hline
\end{tabular}

For feminine head nouns, the sequence in which gender is held constant and only number enters into agreement attraction (F.SG F.PL F.PL) was rated significantly higher than the sequence in which both number and gen- 
Table 6. Potential attraction conditions with masculine head nouns

\begin{tabular}{ccc|cc}
\hline NP1 & NP2 & ADJ & Rating & SE \\
\hline M.SG & M.PL & M.PL & 3.58 & 0.27 \\
M.SG & F.PL & F.PL & 3.37 & 0.23 \\
\hline
\end{tabular}

der enter into agreement attraction (F.SG M.PL M.PL; $t=-1.91, \mathrm{p}<0.05$ ). A Wilcoxon test found no significant difference in the processing times for these two conditions (F.SG F.PL F.PL reaction time: $1905 \mathrm{~ms}$; F.SG M.PL M.PL reaction time: $2007 \mathrm{~ms}$ ). For masculine head nouns, the difference between ratings given for single-error attraction conditions (i.e., M.SG M.PL M.PL) and double-error attraction conditions (i.e., M.SG F.PL F.PL) was not significant $(\mathrm{p}=0.48)$, though we note the trend suggesting double errors receive lower ratings. A Wilcoxon test also found no significnt differece in the processing times for these two conditions (M.SG M.PL M.PL reaction time: $2014 \mathrm{~ms}$; M.SG F.PL F.PL: 2440 ms.).

\section{Internal structure of Spanish number and gender}

In the initial predictions, we stated that if a feature value is specified, then we should see relevant grammaticality effects. In other words, speakers should be able to distinguish between sentences in which agreement between the head noun and adjective in this feature value is successful, and ones in which there is an agreement error pertaining to the given feature value. It follows that if a feature is multi-valued, both of its values will exhibit grammaticality effects. If, however, the feature is single-valued, only one of the features might exhibit grammaticality effects.

The results presented in section 3 show that both the singular feature value and the plural feature value for number yield grammaticality effects. Errors in number between the head noun and adjective were rightly perceived as incorrect, and the corresponding sentences were rated significantly lower than sentences in which there was no error in number agreement. Recall the predictions made in section 3.3 :

Prediction 1: If number is multi-valued, both singular and plural will show grammaticality effects. 
Prediction 2: If number is single-valued, either singular or plural (but not both) will show grammaticality effects.

Further, recall our discussion of the possible feature content of Spanish number in section 2.1, which gave conflicting evidence as to whether the singular or plural is specified in Spanish. As in English, the Spanish plural is morphologically specified with $-s$, whereas the singular is morphologically unspecified. However, the Taghlīb Test and coordination facts showed the singular to be semantically specified, and the plural to be semantically unspecified (again just like in English). Based on our results, we conclude that number in Spanish is multi-valued: both singular and plural features are available in Spanish.

The picture for gender is different. Sentences with masculine head nouns exhibit grammaticality effects, while sentences with feminine head nouns do not. Recall the predictions made in section 3.3 (repeated below).

Prediction 3: If gender is multi-valued, both masculine and feminine will show grammaticality effects.

Prediction 4: If gender is single-valued, either masculine or feminine (but not both) will show grammaticality effects.

In order to determine whether feminine or masculine is the specified value in Spanish gender, we consider how masculine and feminine adjectives enter into agreement with nouns of different gender. In our experiment, sentences in which the adjective inside the small clause was masculine were all rated approximately equally high, regardless of whether the head noun was masculine or feminine. In other words, there was an absence of grammaticality effects with feminine head nouns, as the masculine adjective agreeing with a feminine noun was not perceived ungrammatical. Where feminine adjectives incorrectly agreed with masculine head nouns, the ungrammaticality was readily detected. This result indicates that masculine adjectives appear not to be concerned with the gender of the head noun, thus confirming that the masculine can be evaluated as the absence of a specified gender, not a gender in its own right.

Feminine adjectives, on the other hand, evoked strong grammaticality effects. Conditions in which feminine adjectives agreed with feminine head nouns (F-F-F and F-M-F) were rated significantly higher than ungrammatical conditions in which the feminine adjective was intended to agree with a masculine head noun (M-F-F and M-M-F). This confirms that the feminine gender categorically excludes masculine from its specification. 
Given the lack of grammaticality effects with masculine predicates, we conclude that Spanish gender is single-valued, and that the feminine is specified while the masculine is the realization of the absence of gender specification. This result is in full support of Harris's analysis according to which the masculine gender in Spanish is the absence of gender specification.

\section{Spanish number and gender: Bundling or splitting?}

Having settled the feature content of number and gender, we can now consider whether the two features are bundled together or split. In the experiment, we systematically manipulated number and gender in agreement attraction conditions in order to create conflict between features and observe how speakers perceive the resulting sentences.

We can compare ratings assigned to sentences in which agreement attraction occurs in both the number and gender with ratings given to sentences in which attraction occurs with just one feature. These conditions are illustrated in (19-a) and (19-b), respectively.
a. *El niño considera la noticia en los
the boy consider.3SG the news.item.F.SG in the periódicos terriblements aburridos.
magazines.M.PL terribly boring.M.PL
b. *El niño considera la noticia en las the boy consider.3SG the news.item.F.SG in the revistas terriblements aburridas. magazines.F.PL terribly boring.F.PL Intended: 'The boy considers the news item in the magazines to be terribly boring.'

Recall the predictions made in section 3.3:

Prediction 5: If number and gender are bundled, agreement attraction in one feature should lead to agreement attraction in the other feature.

Prediction 6: If number and gender are split, agreement attraction in one feature need not lead to agreement attraction in the other feature. 
If number and gender are bundled, and therefore valued, together, both of the above sentences should be rated equally high (perhaps mistakenly so, as in cases of attraction). On the other hand, if number and gender are split, then speakers may perceive sentences in which there are two agreement errors as less grammatical than ones in which there is only one agreement error.

As we saw, speakers rated these two attraction cases differently: sentences with just number entering into attraction were rated higher than sentences with both number and gender entering into attraction (cf. Tables 5 and 6). Likewise, we find a trend such that sentences where agreement attraction was caused by gender features alone were rated higher than those with both number and gender causing attraction effects. This suggests that the violation in two features creates a cumulative effect and leads to degradation in ratings, a finding that is consistent with the results of some recent work on grammatical violations in other linguistic domains. For example, Haegeman et al. (2014) present and analyze cumulative effects in what has been traditionally assumed as a homogenous phenomenon of subject island violations. It remains to be seen what other phenomena could be accounted for in terms of cumulative violation effects.

The presence of cumulative errors (or lack of bundled attraction) may not be sufficient to discount the bundling model. To give this model yet another chance, let's consider the following metaphor. A florist is putting together a set of arrangements, where all the roses are red. She accidentally leaves a couple of white roses in one of the arrangements, and the person inspecting the arrangements notices that. The flowers are still all bundled together, but that does not prevent noticing a violation in the color scheme. One could take our gender and number results and interpret them the same way; every time there is a violation, the comprehenders rate the sentences lower, yet that does not mean they access number and gender separately. On this account, however, we would expect that violations in multi-valued features would be rated the same; to continue with our metaphor, it does not matter if our florist forgot to remove a white rose or a pink rose from the bundle, all that matters is that the red color scheme is disrupted. Recall that we determined that number is multi-valued, so it provides us with a useful test case. If all that matters is the presence or absence of a violation, regardless of the color or flavor of that violation, we would expect mismatches in number agreement to be rated the same whether it is a mismatch between the singular probe and the plural goal, or the other way around. However, the results point to a different picture; there is a difference in ratings of the singular goal and plural probe (3.77, see Table 2$)$, and the plural goal and singular probe (3.92, 
see Table 1). This indicates that our flower arrangement model, which would allow us to keep all the features still bundled together, is unlikely.

The end result, then, is that the asymmetry in the ratings of agreement mismatches suggests that number and gender are valued separately; were they valued together, we should have found no difference between the conditions in which only one feature determined attraction effects and the conditions where both features caused attraction.

\section{Conclusions and outstanding questions}

The goal of this paper was to test the hierarchical organization of number and gender in Spanish. Both primary data and the experimental work presented here indicate that Spanish number is a represented by a binary (multi-valued) opposition, with singular and plural both specified and equally visible in the feature specification space. With respect to gender, we find that only the feminine is specified, and masculine is simply the absence of gender. This result, supported by our experimental investigation, is consistent with earlier experimental work on Spanish (Vigliocco et al., 1996; Antón-Méndez, 1999; Antón-Méndez et al., 2002; Alcocer and Phillips, 2009) and Italian (Carminati, 2005) and lends novel support to the conception from Harris (1991) that Spanish gender is organized as a privative opposition.

Based on the established feature specifications of number and gender, we have used an attraction experiment to determine whether number and gender belong to the same category space in the phi-feature hierarchy, or whether one feature dominates the other. These two analytical possibilities correspond to the bundling model of gender and number on the one hand, and the split model on the other. Our results show that Spanish number and gender reveal different patterns with respect to agreement attraction, which suggests that they are not equal in the phi-feature hierarchy. On the assumption that agreement in an XP occurs with the highest phi-feature (Matushansky, 2013; Preminger, 2014), we see stronger effects with number agreement, which indicates that number outranks gender on the phi-feature hierarchy. The two features are accessed differently, and the violations in gender are treated differently than violations in number. Furthermore, the presence of violations in both number and gender results in a cumulative effect; comprehenders assess such violations as significantly more offensive than violations in one feature. All factors being equal, this result constitutes an argument in favor of the split model. 
Throughout this paper, we presented the two models as mutually exclusive, and we have attempted to show that the split model is superior in handling the agreement facts in Spanish. However, its success in Spanish does not yet mean that the bundling model is not applicable outside of Spanish. It may well be that both models have citizenship status in linguistic theory, and that they may apply in different languages, as a reflection of parametric variation. Before closing, we would like to offer some preliminary considerations concerning such parametric variation and the corresponding division of labor between the models.

In Spanish, as we saw, number and gender are constructed and accessed independently in the morphology, which is consistent with the split model. However, it is also possible to imagine a language where the access to all gender features or a subset of those features is via number; in such a language, it is conceivable that gender and number would be bundled together. Romanian may be an example of such a language (Bateman and Polinsky, 2010). In the singular, Romanian distinguishes two noun classes, feminine and the rest (traditional accounts posit three genders, feminine, neuter, and masculine, but neuter and masculine are indistinguishable in terms of their endings and determine the same agreement). In the plural, there are also two classes. However, short of the small, semantically determined number of items whose natural gender is reflected in their grammatical gender, the two-way gender distinction in the plural is predicted from the form of the plural, not from the form of the singular or from independent gender information (Bateman and Polinsky, 2010, pp. 63-64). A Romanian speaker needs the rules of plural formation independent of gender, and can then use the information on the plural to predict agreement class, that is gender. Given that gender appears to get accessed via number, it is conceivable that such a system would be best captured by the bundling model, although it remains to be seen if such a model can be motivated for the singular as well.

A final note: We considered only the gender on inanimate nouns. Our results therefore point to the structural representation of categories within a noun phrase in (20), with gender arguably represented on the nominal root or on $n$. Such a representation effectively reflects the observation that nouns are lexically specified for gender in the lexicon, either at the root level, as shown in (20-a), or as property of $n$, as shown in (20-b). The data that we have been using do not allow us to distinguish between the two models, and we remain agnostic as to which of them is correct.

$$
\begin{aligned}
& \text { a. }\left[\mathrm{DP} \ldots\left[\mathrm{NumP} \ldots\left[\mathrm{NP} \ldots\left[n \mathrm{n} \ldots\left[\sqrt{\mathrm{P}}_{\{\text {Gender }\}}\right]\right]\right]\right]\right] \\
& \text { b. }\left[\mathrm{DP} \ldots\left[\mathrm{NumP} \cdots\left[\mathrm{NP} \cdots\left[{ }_{n \mathrm{P}}\{\text { Gender }\}[\sqrt{\mathrm{P}}]\right]\right]\right]\right]
\end{aligned}
$$


If we now add animate and human nouns, whose gender is often specified, evidence emerges in support of what has been known as the Distributed Gender Hypothesis (Kramer, 2013; Steriopolo and Wiltschko, 2008): there are at least two gender features, a feature for natural gender, projected at the periphery of DP, and grammatical gender, projected below the N-level (either as a property of $n$ or as property of roots). An obvious extension of the study presented here is to test whether natural gender, which is arguably projected at the DP periphery, causes stronger attraction effects as compared to (a) lexical gender and (b) number (see Antón-Méndez et al. 2002 for a discussion of natural vs. lexical gender in relation to number). It is not inconceivable that the projection of natural gender could dominate the projection of number; if so, we would expect to see reflexes of that arrangement in an experimental setting.

Acknowledgement: Work on this project was supported in part by grants from CASL (Center for Advanced Study of Language) at the University of Maryland and from the Observatory of the Spanish Language in the U.S. at Harvard University to Maria Polinsky. Any opinion, findings, conclusions or recommendations expressed in this material are those of the authors. We would like to thank Giuli Dussias, Ruth Kramer, Nicholas Longenbaugh, Maria Luisa Parra, Nina Radkevich, Allison Ray, Andrés Sanín, and the audience at GLOW 37 in Brussels for helpful comments on this project.

\section{References}

Alarcón, I. (2006). The Second Language Acquisition of Spanish Gender Agreement: The Effects of Linguistic Variables on Accuracy. Munich: Lincom Europa.

Alcocer, P. and C. Phillips (2009). A cross-language reversal in illusory agreement licensing. Poster presented at the 22nd annual CUNY conference on human sentence processing, University of California, Davis.

Antón-Méndez, I., J. L. Nicol, and M. F. Garrett (2002). The relation between gender and number agreement processing. Syntax 5, 1-25.

Antón-Méndez, M. I. (1999). Gender and Number: Agreement Processing in Spanish. Ph. D. thesis, University of Arizona.

Baker, M. (2008). The Syntax of Agreement and Concord. Cambridge: Cambridge University Press.

Bateman, N. and M. Polinsky (2010). Romanian as a two-gender language. In D. Gerdts, J. Moore, and M. Polinsky (Eds.), Hypothesis A/Hypothesis B, pp. 41-77. Cambridge, MA: MIT Press. 
Becker, M. and J. Levine (2010). Experigen: An online experiment platform. Available at https://github.com/tlozoot/experigen.

Bobaljik, J. (2008). Where's phi? Agreement as a post-syntactic operation. In D. Harbour, D. Adger, and S. Béjar (Eds.), Phi-Theory: Phi Features across Interfaces and Modules, pp. 295-328. Oxford: Oxford University Press.

Bobaljik, J. and C. Zocca (2011). Gender markedness: The anatomy of a counter-example. Morphology 21, 141-166.

Bock, K., M. Carreiras, and E. Meseguer (2012). Number meaning and number agreement in English and Spanish. Journal of Memory and Language 66, 17-37.

Bock, K. and J. C. Cutting (1992). Regulating mental energy: Performance units in langauge production. Journal of Memory and Language 31, 99-127.

Bock, K. and K. Eberhard (1993). Meaning, sound, and syntax in English number agreement. Language and Cognitive Processes 8, 57-99.

Bock, K., K. Eberhard, J. C. Cutting, A. Meyer, and H. Schriefers (2001). Some attractions of verb agreement. Cognitive Psychology 43, 83-128.

Bock, K. and C. A. Miller (1991). Broken agreement. Cognitive Psychology 23, 45-93.

Bull, W. (1965). Spanish for Teachers: Applied Linguistics. New York: Ronald Press.

Carminati, M. N. (2005). Processing reflexes of the Feature Hierarchy (person $>$ number $>$ gender) and implications for linguistic theory. Lingua 115, 259-285.

Carstens, V. (2000). Concord in minimalist theory. Linguistic Inquiry 31, 319-355.

Carstens, V. (2003). Rethinking complementizer agreement: Agree with a case-checked goal. Linguistic Inquiry 34, 393-412.

Chomsky, N. (1995). The Minimalist Program. Cambridge, MA: MIT Press.

Contreras, H. (1987). Small clauses in Spanish and English. Natural Language \& Linguistic Theory 5, 225-243.

Demonte, V. (1988). Remarks on secondary predicates: C-command, extraction and reanalysis. The Linguistic Review 6, 1-39.

Domínguez, A., F. Cuetos, and J. Segui (1999). The processing of grammatical gender and number in Spanish. Journal of Psycholinguistic Research 28, 485-498.

Franck, J., G. Lassi, U. H. Frauenfelder, and L. Rizzi (2006). Agreement and movement: A syntactic analysis of attraction. Cognition 101, 173-216.

Franck, J., G. Vigliocco, and J. Nicol (2002). Subject-verb agreement errors in French and English: The role of syntactic hierarchy. Language and Cognitive Processes 17, 371404.

Greenberg, J. (1966). Language Universals. The Hague: Mouton.

Haegeman, L., Ángel. L. Jiménez-Fernández, and A. Radford (2014). Deconstructing the subject condition in terms of cumulative constraint violation. The Linguistic Review 31, 73-150.

Harley, H. and E. Ritter (2002). Person and number in pronouns: A feature-geometric analysis. Language $78,482-526$.

Harris, J. (1991). The exponence of gender in Spanish. Linguistic Inquiry 22, 27-62.

Hartsuiker, R., H. Schriefers, K. Bock, and G. Kikstra (2003). Morphophonological influences on the construction of subject-verb agreement. Memory and Cognition 31, $1316-1326$.

Jiménez-Fernández, Ángel. and V. Spyropoulos (2013). Feature inheritance, vP phrases and the information structure of small clauses. Studia Linguistica 67, 185-224. 
Kluender, R. (2004). Are subject islands subject to a processing constraint? In V. Chand, A. Kelleher, A. J. Rodriguez, and B. Schmeiser (Eds.), West Coast Conference on Formal Linguistics (WCCFL) 23, Somerville, MA, pp. 101-125. Cascadilla Press.

Kramer, R. (2009). Definite Markers, Phi-features, and Agreement: A Morphosyntactic Investigation of the Amharic DP. Ph. D. thesis, University of California, Santa Cruz.

Kramer, R. (2013). Gender in Amharic: A morphosyntactic approach to natural and grammatical gender. Language Sciences 43, 102-115.

Levelt, W. J. M. (1989). Speaking: From Intention to Articulation. Cambridge, MA: MIT Press.

Lorimor, H., K. Bock, E. Zalkind, A. Sheyman, and R. Beard (2008). Agreement and attraction in Russian. Language and Cognitive Processes 23, 769-799.

Matushansky, O. (2013). Gender confusion. In L.-S. Cheng and N. Corver (Eds.), Diagnosing Syntax, pp. 271-294. Oxford: Oxford University Press.

Pesetsky, D. and E. Torrego (2007). The syntax of valuation and the interpretability of features. In S. Karimi, V. Samiian, and W. K. Wilkins (Eds.), Phrasal and Clausal Architecture: Syntactic Derivation and Interpretation, pp. 262-294. Amsterdam: John Benjamins.

Phillips, C. (2013). Some arguments and non-arguments for reductionist accounts of syntactic phenomena. Language and Cognitive Processes 28, 156-187.

Picallo, C. (1991). Nominals and nominalization in Catalan. Probus 3, 279-316.

Pillot, C., G. Scontras, and L. Clemens (2012). ExperigenRT: Measure reaction times in web-based auditory experiments. Available online at http://pollab.fas.harvard.edu/?q=node/212.

Preminger, O. (2014). Agreement and Its Failures. Cambridge, MA: MIT Press.

Ritter, E. (1993). Where's gender? Linguistic Inquiry 24, 795-803.

Roca, I. M. (1989). The organisation of grammatical gender. Transactions of the Philological Society $87,1-32$.

Sauerland, U. (2003). A new semantics for number. In R. B. Young and Y. Zhou (Eds.), Semantics and Linguistic Theory (SALT) 13, Ithaca, NY, pp. 258-275. CLC Publications.

Sauerland, U., J. Anderssen, and K. Yatsushiro (2005). The plural is semantically unmarked. In S. Kesper and M. Reis (Eds.), Linguistic Evidence: Empirical, Theoretical and Computational Perspectives. Berlin: Mouton de Gruyter.

Scontras, G. (2013a). Accounting for counting: A unified semantics for measure terms and classifiers. In T. Snider (Ed.), Proceedings of SALT 23, pp. 549-569.

Scontras, G. (2013b). A unified semantics for number marking, numerals, and nominal structure. In E. Chemla, V. Homer, and G. Winterstein (Eds.), Proceedings of Sinn und Bedeutung 17, pp. 545-562.

Scontras, G., M. Polinsky, and Z. Fuchs (2013). Agreement mismatches in Spanish. http://hdl.handle.net/1902.1/21954; Harvard Dataverse Network V3.

Steriopolo, O. and M. Wiltschko (2008). Distributed GENDER hypothesis. Paper presented at FDSL 7.5, Independent University of Moscow.

Vigliocco, G., B. Butterworth, and M. F. Garrett (1996). Subject-verb agreement in Spanish and English: Differences in the role of conceptual constraints. Cognition 61, 261-298.

Vigliocco, G., B. Butterworth, and C. Semenza (1995). Constructing subject-verb agreement in speech: The role of semantic and morphological factors. Journal of Memory and Language 34, 186-215. 
Vigliocco, G. and J. Franck (1999). When sex and syntax go hand in hand: Gender agreement and langauge production. Journal of Memory and Language 40, 455-478.

Vigliocco, G. and J. Nicol (1998). Separating hierarchical relations and word order in language production: Is proximity concord syntactic or linear? Cognition 68, B13-B29.

Warren, T. and E. Gibson (2002). The influence of referential processing on sentence complexity. Cognition 85, 79-112.

Wechsler, S. and L. Zlatić (2003). The Many Faces of Agreement. Stanford, CA: CSLI. 